\title{
Isotonic $M$-Estimation of Location: Union-Intersection Principle and Preliminary Test Versions
}

\author{
Azza R. Karmous \\ Zagazig University, Zagazig, Egypt \\ AND \\ Pranab K. Sen* \\ University of North Carolina, Chapel Hill, North Carolina \\ Communicated by the Editors \\ In a $k(\geqslant 2)$ sample model, isotonic estimators of locations $\theta_{1}, \ldots, \theta_{k}$ take into \\ consideration the prior restraint that $\theta_{1} \leqslant \cdots \leqslant \theta_{k}$. Though these estimators are \\ appealling, they are generally biased. The union-intersection (UI-) principle and \\ the theory of $M$-estimation of location are incorporated in the formulation of some \\ robust, preliminary test, isotonic $(M-)$ estimators of locations. Associated \\ distribution theory of the test statistic and estimator is studied in a systematic
} manner. (C) 1988 Academic Press, Inc

\section{INTRODUCTION}

Let $X_{i j}, j=1, \ldots, n_{i}$ be $n_{i}$ independent and identically distributed random variables (i.i.d.r.v.) with a distribution function (d.f.) $F_{i}$, defined on the real linc $R$, for $i=1, \ldots, k(\geqslant 2)$; all these $k$ samples are assumed to be independent. Consider the usual location model

$$
F_{i}(x)=F\left(x-\theta_{i}\right), \quad i=1, \ldots, k,
$$

Received December 11, 1987.

AMS 1980 subject classifications: 62F35, 62G05, $62 \mathrm{G} 99$.

Key words and phrases: (isotonic) $M$-estimators, ordered and orthant alternatives, preliminary $M$-test, preliminary test estimator, UI-principle.

* Work of this author was partially supported by the Office of Naval Research, Contract N000i4-83-K-0387. 
where the $\theta_{i}$ are the location parameters and $F$ is a continuous d.f., assumed to be symmetric about 0 . It is desired to develop suitable $M$-estimators of the vector of location parameters $\theta=\left(\theta_{1}, \ldots, \theta_{k}\right)^{\prime}$ following a preliminary test of

$$
H_{0}: \theta_{1}=\cdots=\theta_{k} \quad \text { against } \quad H_{1}: \theta_{1} \leqslant \cdots \leqslant \theta_{k} \text {, }
$$

where at least one of the inequalities is strict. The preliminary $M$-test is an extension of union-intersection (UI-) tests considered by De [5], Chinchilli and Sen [3,4], and Boyd and Sen [2], while the preliminary test estimator (PTE) is formulated along the lines of Sen and Saleh [10], but for restricted alternatives.

Section 2 deals (succinctly) with the classical $M$-estimators of location in this multi-sample context; the corresponding UI- $M$-test for $H_{0}$ against $H_{1}$ is considered in Section 3. The results of Section 2 and 3 are incorporated in the formulation of isotonic $M$-estimators and their PTE versions in Sections 4 and 5, respectively. Asymptotic properties of these estimators are studied under a sequence of local alternatives containing $H_{0}$ as a special case. The concluding section deals with this relative picture through some simulation studies.

\section{2. $M$-Estimators of Location and RegUlaRity CONDITIONS}

We introduce first a score function $\psi: R \rightarrow R$, defined by

$$
\psi(x)=\psi_{1}(x)+\psi_{2}(x), \quad x \in R=(-\infty, \infty),
$$

where both $\psi_{1}$ and $\psi_{2}$ are nondecreasing and skew-symmetric functions with $\psi_{1}$ absolutely continuous on any bounded interval in $R$ and $\psi_{2}$ a step function having finitely many jumps. We denote these jump-points by $-\infty=a_{0}<a_{1}<\cdots<a_{p}<a_{p+1}=\infty$ and assume that there exist real numbers $\alpha_{0}<\cdots<\alpha_{p}$, such that $\psi_{2}(x)=\alpha_{j}$, for $x \in\left(a_{j}, a_{j+1}\right), j=0,1, \ldots, p$, and, conventionally, we let $\psi_{2}\left(a_{j+1}\right)=\left(\alpha_{j}+\alpha_{j+1}\right) / 2$, for $j=0, \ldots, p-1$. We assume that

$$
0<\sigma_{\psi}^{2}=\int_{R} \psi^{2}(x) d F(x)<\infty,
$$

and

$\int_{R}\left\{\psi_{1}^{\prime}(x)\right\}^{2} d F(x)<\infty, \quad$ where $\quad \psi_{1}^{\prime}(x)=(d / d x) \psi_{1}(x), \quad x \in R$. 
Concerning the d.f. $F$, we assume that it has an absolutely continuous density function $f$, such that $f^{\prime}(x)=(d / d x) f(x)$ exists almost everywhere (a.e.), and that

$\mathscr{I}(f)=\int_{R}\left\{f^{\prime}(x) / f(x)\right\}^{2} d F(x)<\infty \quad$ (i.e., finite Fisher information).

These regularity conditions are all adapted from Jurečková [7].

Now, for each $i(=1, \ldots, k)$ and every real $t$, we define

$$
M_{i, n_{i}}(t)=\sum_{j=1}^{n_{i}} \psi\left(X_{i j}-t\right), \quad t \in R,
$$

and note that by definition $M_{i, n_{i}}(t)$ is $\searrow$ in $t \in R$. Let then

$$
\begin{gathered}
\hat{\theta}_{i, n_{i}}^{(1)}=\sup \left\{t: M_{i, n_{i}}(t)>0\right\}, \quad \hat{\theta}_{i, n_{i}}^{(2)}=\inf \left\{t: M_{i, n_{i}}(t)<0\right\} ; \\
\hat{\theta}_{i, n_{i}}=\left(\hat{\theta}_{i, n_{i}}^{(1)}+\hat{\theta}_{i, n_{i}}^{(2)}\right) / 2, \quad i=1, \ldots, k ; \quad \hat{\boldsymbol{\theta}}_{n}=\left(\hat{\theta}_{\left.1, n_{1}, \ldots, \hat{\theta}_{k, n_{k}}\right)^{\prime} .} .\right.
\end{gathered}
$$

Then, $\hat{\boldsymbol{\theta}}_{n}$ is the vector of $M$-estimators of location parameters based on the common score function $\psi$. In this context, recall that the assumed symmetry of $F$ and the skew-symmetry of $\psi$ (around 0 ) imply that $\bar{\psi}=\int_{R} \psi(x) d F(x)=0$, and this motivates the normal equations in (2.6) - (2.7) for the solution of the $M$-estimators. These $M$-estimators are translation-equivariant, and depending on the choice of the score function $\psi$, they are robust too. For later use, we present the following asymptotic results.

First, it follows from Jurečková [7] and Singer and Sen [11] that for any (fixed) $T: 0<T<\infty$, for each $i(=1, \ldots, k)$, as $n_{i} \rightarrow \infty$,

$$
\sup \left\{n_{i}^{-1 / 2}\left|M_{i, n_{i}}\left(\theta_{i}+n_{i}^{-1 / 2} t\right)-M_{i, n_{i}}\left(\theta_{i}\right)+n_{i}^{1 / 2} \gamma t\right|:|t| \leqslant T\right\} \stackrel{P}{\longrightarrow} 0,
$$

where

$$
\gamma=\int_{R} \psi(x)\left\{-f^{\prime}(x) / f(x)\right\} d F(x) \text { is finite and positive. }
$$

We let $n=n_{1}+\cdots+n_{k}$ and assume that as $n$ increases,

$$
n_{i} / n \rightarrow \lambda_{i}: 0<\lambda_{i}<1, \quad \text { for each } i(=1, \ldots, k) ; \quad \sum_{i=1}^{k} \lambda_{i}=1 .
$$

A direct consequence of (2.8), (2.9), (2.10), and the asymptotic normality of the $M$-statistics (studied in detail in Jurečková [7]) is the following: As $n \rightarrow \infty$,

$$
n^{1 / 2}\left(\hat{\boldsymbol{\theta}}_{n}-\boldsymbol{\theta}\right) \stackrel{\mathscr{Q}}{\longrightarrow} \mathcal{N}_{k}\left(\mathbf{0}, \gamma^{-2} \sigma_{\psi}^{2} \Lambda^{-1}\right) ; \quad \boldsymbol{\Lambda}=\operatorname{Diag}\left(\lambda_{1}, \ldots, \lambda_{k}\right) .
$$


Finally, (2.11) ensures that

$$
n^{1 / 2}\left\|\hat{\boldsymbol{\theta}}_{n}-\boldsymbol{\theta}\right\|=O_{p}(1) \quad \text { (i.e., bounded in probability) }
$$

\section{The UI-PreliminaRY $M$-TesT}

Making use of (2.11), we shall incorporate the UI-principle and extend the classical test of Barlow et al. [1] to general $M$-statistics. Let

$\omega=\left\{\boldsymbol{\theta}: \theta_{1}=\cdots=\theta_{k}=\theta \in R\right\} \quad$ and $\quad \omega^{*}=\left\{\boldsymbol{\theta}: \theta_{1} \leqslant \cdots \leqslant \theta_{k}\right\}$.

The (approximate) likelihood function of $\hat{\boldsymbol{\theta}}_{n}$ is given by

$$
\left.L_{n}(\boldsymbol{\theta})=\prod_{i=1}^{k}\left\{\gamma^{2} n_{i} / 2 \pi \sigma_{\psi}^{2}\right)^{1 / 2} \exp \left[-n_{i}\left(\hat{\theta}_{i, n_{i}}-\theta_{i}\right)^{2} \gamma^{2} / 2 \sigma_{\psi}^{2}\right]\right\} .
$$

Therefore, we have

$$
\sup \left\{L_{n}(\boldsymbol{\theta}): \boldsymbol{\theta} \in \omega\right\}=\mathrm{const}\left\{\exp \left[-\left(\gamma^{2} / 2 \sigma_{\psi}^{2}\right) \sum_{i=1}^{k} n_{i}\left(\hat{\theta}_{i, n_{i}}-\widetilde{\theta}_{n}\right)^{2}\right]\right\}
$$

where

$$
\tilde{\theta}_{n}=\sum_{i=1}^{k}\left(n_{i} / n\right) \hat{\theta}_{i, n_{i}}
$$

In passing, we may remark that under $\omega$, a natural estimator of $\theta$ might have been obtained by equating $\sum_{i=1}^{k} M_{i, n_{i}}(t)$ to 0 (in the same fashion as in (2.6)-(2.7)). In view of (2.8), this natural estimator of $\theta$ would be squareroot $n$ equivalent (in probability) to $\ddot{\theta}_{n}$. From the computational point of view, given the individual sample $M$-estimators in (2.6)-(2.7), (3.4) involves no extra computation, while the computation of the natural estimator is certainly more involved (although a few iterations should give the estimator up to any desired degree of accuracy). From the point of view of robustness, for small or moderate sample sizes, the natural estimator has some advantage, although in the aymptotic case, there is hardly any difference. Next, we note that

$\omega^{*}=\bigcup_{\mathbf{a} \in A} \omega(\mathbf{a}) ; \quad \omega(\mathbf{a})=\left\{\boldsymbol{\theta}: \theta_{i}=\theta+\delta a_{i}, 1 \leqslant i \leqslant k, a_{1} \leqslant \cdots \leqslant a_{k}\right\}$,

where $\delta$ is a positive scalar constant, and a belongs to a positively homogeneous cone $\mathscr{A}$. Without any loss of generality, we may set $\bar{a}=n^{-1} \sum_{i=1}^{k} n_{i} a_{i}=0$ and $\sum_{i=1}^{k} n_{i} a_{i}^{2}=n$. 
Under $\omega(\mathbf{a})$, based on (3.2), the MLE of $\delta$ and $\theta$ are given by

$$
\begin{gathered}
\delta_{n}^{*}(\mathbf{a})=\sum_{i=1}^{k} \lambda_{i} a_{i} \hat{\theta}_{i, n_{i}} I\left(\sum_{i=1}^{k} n_{i} a_{i} \hat{\theta}_{i, n_{i}} \geqslant 0\right), \\
0_{n}^{*}=\sum_{i=1}^{k}\left(n_{i} / n\right) \hat{\theta}_{i, n_{i}}=\partial_{n},
\end{gathered}
$$

where $I(A)$ stands for the indicator function of the set $A$. Substituting (3.6) in (3.2) and using (3.3) and (3.4), we obtain that

$$
\begin{aligned}
L_{n}(\mathbf{a}) & =-2 \log \left\{\left[\sup \left\{L_{n}(\boldsymbol{\theta}): \boldsymbol{\theta} \in \omega\right\}\right] /\left[\sup \left\{L_{n}(\boldsymbol{\theta}): \boldsymbol{\theta} \in \omega(\mathbf{a})\right\}\right]\right\} \\
& =n\left(\gamma^{2} / \sigma_{\psi}^{2}\right)\left\{\sum_{i=1}^{k} \lambda_{i} a_{i}\left(\hat{0}_{i, n_{i}}-\partial_{n}\right)\right\}^{2} \cdot I\left(\sum_{i=1}^{k} n_{i} a_{i} \hat{\theta}_{i, n_{i}} \geqslant 0\right) .
\end{aligned}
$$

We reject the null hypothesis $H_{0}$ in favor of $\omega(\mathbf{a})$ for large values of $L_{n}(\mathbf{a})$. To obtain an overall test for the entire alternative $\omega=\bigcup_{\mathbf{a} \in A} \omega(\mathbf{a})$, we incorporate the Roy UI-principle, so that on letting

$$
\mathscr{A}=\left\{\mathbf{a}: a_{1} \leqslant \cdots \leqslant a_{k}, \bar{a}=0 \text { and } \sum_{i=1}^{k} \lambda_{i} a_{i}^{2}=1\right\} \text {, }
$$

we set the UI-test statistic as

$$
L_{n}^{*}=\sup \left\{L_{n}(\mathbf{a}): \mathbf{a} \in \mathscr{A}\right\} .
$$

Our main task is to derive a simple asymptotic expression for $L_{n}^{*}$ and to study its distribution theory (under the null as well as local alternatives). Towards this venture, we make use of the basic results in (2.8) through (2.12) along with the Kuhn-Tucker-Lagrange (KTL-) point formula theorem in the nonlinear programming theory. We consider a sequence $\left\{H_{n}\right\}$ of local alternatives

$$
H_{n}: \boldsymbol{\theta}=\boldsymbol{\theta}_{(n)}=\theta \mathbf{1}+n^{-1 / 2} \xi, \quad \xi \in \mathscr{A}, \quad \text { so that } \lambda^{\prime} \xi=0,
$$

where $\lambda=\left(\lambda_{1}, \ldots, \lambda_{k}\right)^{\prime}$ and $\xi$ is an arbitrary (fixed) vector in $\mathscr{A}$. By an appeal to (2.12) and (3.4), we obtain that under (3.10), $n^{1 / 2}\left|\tilde{\theta}_{n}-\theta\right|=O_{p}(1)$, and hence, by $(2.8)$, we obtain that for each $i(=1, \ldots, k)$, as $n \rightarrow \infty$,

$$
\begin{gathered}
n^{-1 / 2}\left[M_{i, n_{i}}\left(\tilde{\theta}_{n}\right)-M_{i, n_{i}}\left(\hat{\theta}_{i, n_{i}}\right)\right]=n^{1 / 2} \gamma \lambda_{i}\left(\hat{\theta}_{i, n_{i}}-\tilde{\theta}_{n}\right)+o_{p}(1), \\
n^{-1 / 2} M_{i, n_{i}}\left(\hat{\theta}_{i, n_{i}}\right)=o_{p}(1), \quad \text { by }(2.5)-(2.8) .
\end{gathered}
$$


Consequently, writing $\hat{M}_{i, n_{i}}=M_{i, n_{i}}\left(\theta_{n}\right)$ (the residual $M$-statistic), $i=1, \ldots, k$; $\hat{\mathbf{M}}_{n}=\left(\hat{M}_{1, n_{1}}, \ldots, \hat{M}_{k, n_{k}}\right)^{\prime}$, we obtain from (2.6), (2.11), and the above relations that under $\left\{H_{n}\right\}$ (as well as $H_{0}$ ),

$$
\begin{gathered}
n^{1 / 2} \gamma \sum_{i=1}^{k} \lambda_{i} a_{i}\left(\hat{\theta}_{i, n_{i}}-\partial_{n}\right)=n^{-1 / 2} \mathbf{a}^{\prime} \hat{\mathbf{M}}_{n}+o_{p}(1) ; \\
n^{-1 / 2} \hat{\mathbf{M}}_{n} \stackrel{\mathscr{P}}{\longrightarrow} \mathscr{N}_{k}\left(\gamma \mathbf{\Lambda} \xi, \sigma_{\psi}^{2}\left(\boldsymbol{\Lambda}-\lambda \lambda^{\prime}\right)\right) .
\end{gathered}
$$

The use of these residual $M$-statistics eliminates the need to estimate the unknown parameter $\gamma$ (for the construction of a suitable test statistic) and also introduces other simplifications to follow. To construct $L_{n}^{*}$ in (3.9), we introduce another reparameterization whereby we reduce the problem to an orthant alternative problem, for which the KTL-point formula works out neatly. Let

$$
\boldsymbol{\beta}=\mathbf{D \theta} \text {, where } \underset{(k-1) \times k}{\mathbf{D}}=\left(\begin{array}{rrrrr}
-1 & 1 & 0 & \cdots & 0 \\
0 & -1 & 1 & \cdots & 0 \\
\cdots & \ldots & \ldots & \ldots & \ldots \\
0 & 0 & \cdots & -1 & 1
\end{array}\right) \text { is of rank } k-1 .
$$

Then (3.5) can equivalently be written as

$$
\omega^{*}=\bigcup_{\mathbf{b} \in B} \omega^{0}(\mathbf{b}) ; \quad \omega^{0}(\mathbf{b})=\left\{\mathbf{b}: b_{j} \geqslant 0, j=2, \ldots, k\right\},
$$

and $B$ is the $(k-1)$-dimensional positive orthant. Let

$$
\tilde{M}_{n, i}=\sum_{j-i}^{k} \hat{M}_{j, n_{j}}, \quad i=2, \ldots, k ; \quad \tilde{\mathbf{M}}_{n}=\left(\tilde{M}_{n, 2}, \ldots, \tilde{M}_{n, k}\right)^{\prime}
$$

Then,

$n^{-1 / 2} \mathbf{a}^{\prime} \hat{\mathbf{M}}_{n}=n^{-1 / 2} \mathbf{b}^{\prime} \tilde{\mathbf{M}}_{n} \quad$ whenever $\quad a_{i}=a_{i-1}+b_{i}, \quad i=2, \ldots, k ;$

$$
n^{-1 / 2} \tilde{\mathbf{M}}_{n} \stackrel{\mathscr{Q}}{\longrightarrow} \mathcal{N}_{k-1}\left(\gamma \mathbf{U} \mathbf{\Lambda} \xi, \sigma_{\psi}^{2} \Lambda^{*}\right) ; \quad \mathbf{U}=\left(\left(u_{j j^{\prime}}\right)\right), \quad \Lambda^{*}=\left(\left(\lambda_{j j^{\prime}}^{*}\right)\right) ;
$$

where

$$
\begin{array}{ll}
u_{j j^{\prime}}=0 & \text { if } j^{\prime}<j \quad \text { and } 1 \quad \text { if } j^{\prime} \geqslant j \quad\left(2 \leqslant j \leqslant k, 1 \leqslant j^{\prime} \leqslant k\right), \\
& \lambda_{j j^{\prime}}^{*}=\sum_{i=j \vee j^{\prime}}^{k} \lambda_{i}-\left(\sum_{i=j}^{k} \lambda_{i}\right)\left(\sum_{i=j^{\prime}}^{k} \lambda_{i}\right), \quad \text { for } j, j^{\prime}=2, \ldots, k .
\end{array}
$$


$\theta_{(n)}$ now belongs to the lower dimensional space in (5.16) (actually the boundary of an $l$-dimensional subset of $\omega^{*}$ ). It is easy to show that under (5.16), $P\left\{\hat{\boldsymbol{\theta}}_{n} \in \boldsymbol{R}_{\pi} \mid \boldsymbol{\theta}_{(n)}\right\}$ converges to 0 as $n$ increases, for every $\pi$ not belonging to $\Pi_{l}$. On the other hand, for $\pi$ belonging to $\Pi_{l}$, $P\left\{n \mathbf{D}_{\pi}\left(\hat{\boldsymbol{\theta}}_{n}-\boldsymbol{\theta}_{(n)}\right) \leqslant \mathbf{x}+\tau^{* 0}, \hat{\boldsymbol{\theta}}_{n} \in R_{\pi} \mid \boldsymbol{\theta}_{(n)}\right\}$ has a nondegenerate limit, where the $\tau^{* 0}$ are defined as in (5.4) with the $\tau_{r}$ being replaced by the $\tau_{r}^{*}$. Thus, under (5.16), the asymptotic distribution function of the IME is given by

$$
\begin{aligned}
\lim _{n \rightarrow \infty} P\left\{n^{1 / 2}\left(\boldsymbol{\theta}_{n}^{*}-\boldsymbol{\theta}_{(n)}\right) \leqslant \mathbf{x} \mid(5.16)\right\} \\
\quad=\lim _{n \rightarrow \infty}\left[\sum_{\pi \in \Pi_{l}} P\left\{n^{1 / 2} \mathbf{D}_{\pi}\left(\hat{\boldsymbol{\theta}}_{n}-\boldsymbol{\theta}_{(n)}\right) \leqslant \mathbf{x}+\tau^{* 0}, \hat{\boldsymbol{\theta}}_{n} \in R_{\pi} \mid(5.16)\right\}\right] .
\end{aligned}
$$

It may be noted that for $l=1, \Pi_{l}=\Pi$ and (5.18) reduces to (5.5), while for $l \geqslant 2,(5.18)$ involves a subset of the terms appearing in (5.5), and hence, the two forms are not isomorphic. In passing, we may remark that if (5.17) holds for $l=k$ then within each of the $k$ buckets, there is only one element, and hence, $\Pi_{k}$ consists of the cone $\hat{\theta}_{1, n_{1}} \leqslant \cdots \leqslant \hat{\theta}_{k, n_{k}}$. As such, (4.5) holds with $\mathbf{D}_{\pi}=\mathbf{I}$ with probability converging to 1 as $n \rightarrow \infty$. Thus, in this case, the classical $M$-estimator and the IME based on the same score function becomes asymptotically equivalent, in probability. Thus, (2.11) applies to the IME as well.

It is quite clear that the computation of the exact bias and mean product matrix of the IME and PTIME is highly involved; even the asymptotic case is not that simple to handle. For small values of $k$ (viz., $k=3,4$, etc.), term by term evaluation of $(5.5)$ or (5.18) is possible, although the task becomes prohibitively laborious as $k$ increases. For this reason, we take recourse to simulation studies of the relative bias and efficiency of the PTIME and IME. In this context, we interpret the relative efficiency $\left(e^{*}\right)$ of the PTIME with respect to the IME in the usual way as the inverse ratio of the generalized variance of their respective asymptotic distributions.

\section{Some Simulation Studies}

We consider specifically the case of three samples (i.e., $k=3$ ) and for the $M$-estimators of location, we choose the Huber score function with $K=1.5$, i.e., we take

$$
\psi(x)= \begin{cases}x, & |x| \leqslant K=1.5 \\ K \operatorname{sign} x, & |x|>K\end{cases}
$$

All the samples are generated by random normal deviates with appropriate shifts in the location parameters. Since the $M$-estimators are translation- 
for $r=0, \ldots, k-1$. These orthant probabilities may be computed by reference to the asymptotic normality result in (3.19) (where under $H_{0}$, $\xi=0$ ) and the tables for the multinormal orthant probabilities considered by Gupta [6] and others. Once these $w_{r}$ are computed, the critical level $c$ for which (3.27) corresponds to $1-\alpha$, for some prespecified level of significance $\alpha(0<\alpha<1)$, can easily be obtained from the tables for the central chi-square distributions, available extensively in the literature. We denote this critical level by $c_{\alpha}^{*}$. Then, the UI-preliminary test for $H_{0}$ vs. $H_{1}$, based on the residual $M$-statistics, may be carried out as follows:

$$
\text { Reject or accept } H_{0} \text { according as } \mathscr{L}_{n}^{*} \text { is } \geqslant \text { or }\left\langle c_{\alpha}^{*}\right. \text {. }
$$

A key factor in the simplification of this asymptotic null distribution of the proposed UI-test statistic is the (asymptotic) independence (for each $J: \phi \subseteq J \subseteq \mathscr{J}$ ) of the quadratic form and the two indicator functions in the right-hand side of (3.25). Unfortunately the non-null distribution (even for local alternatives) is not expressible in terms of averages of appropriate non-central chi-squared distributions. This problem arises mainly due to the fact that when the null hypothesis is not true, though $\Lambda_{\left(J^{\prime} J^{\prime}\right)}^{*} \tilde{\mathbf{M}}_{n\left(J^{\prime}\right)}$ in (3.25) is (asymptotically) independent of $\tilde{\mathbf{M}}_{n\left(J: J^{\prime}\right)}$ and the quadratic form $\tilde{\mathbf{M}}_{n\left(J: J^{\prime}\right)}^{\prime} \boldsymbol{\Lambda}_{\left(J J: J^{\prime}\right)}^{*-1} \tilde{\mathbf{M}}_{n\left(J: I^{\prime}\right)}$, the later random variable is not independent of $I\left(\tilde{\mathbf{M}}_{n\left(J: J^{\prime}\right)} \geqslant \mathbf{0}\right)$. As such, the best we can do is to express the asymptotic non-null distribution of $\mathscr{L}_{n}^{*}$, under $\left\{H_{n}\right\}$, in the form

$$
\begin{aligned}
P\left\{\mathscr{L}_{n}^{*} \leqslant c \mid H_{n}\right\} \sim & \sum_{\phi \subseteq J \subseteq \mathscr{g}} P\left\{\tilde{\mathbf{M}}_{n\left(J: J^{\prime}\right)} \boldsymbol{\Lambda}_{\left(J J: J^{\prime}\right)}^{*-1} \tilde{\mathbf{M}}_{n\left(J: J^{\prime}\right)}\right. \\
\leqslant & \left.n c \sigma_{\psi}^{2}, \tilde{\mathbf{M}}_{n\left(J: J^{\prime}\right)} \geqslant \mathbf{0} \mid H_{n}\right\} \\
& \times P\left\{\boldsymbol{\Lambda}_{\left(J^{\prime} J^{\prime}\right)}^{*-1} \tilde{\mathbf{M}}_{n\left(J^{\prime}\right)} \leqslant \mathbf{0} \mid H_{n}\right\} .
\end{aligned}
$$

For the right-hand side, the second factor can be evaluated using the normal orthant probability tables, but for appropriate shifts, while evaluation of the first factor may be quite involved. Though the non-central chi-square (bar) distribution may not generally hold for (3.30), there are alternative forms involving central chi-square distributions with mixing coefficients depending on the alternative hypothesis which have been worked out by some authors (viz., Tsai and Sen [12]), and these may be used (to a limited extent) to study the asymptotic power properties of the UI-test. Equation (3.30) is quite amenable for simulation studies of the asymptotic power function, and for some numerical results, we may refer to Karmous [8]. 


\section{IsOTONIC $M$-ESTimation of LoCATION}

We may refer to Barlow et al. [1] for an excellent account of iotonic estimation of the multi-sample normal mean problem. Borrowing their general line of attack and the basic philosophy of $M$-estimation theory, we may present isotonic $M$-estimators of the location vector $\theta$ as the solution of

$$
\begin{gathered}
\sum_{i=1}^{k}\left|\sum_{j=1}^{n_{i}} \psi\left(X_{i j}-\theta_{i}\right)\right| \quad\left(\text { or } \sum_{i=1}^{k}\left[\sum_{j=1}^{n_{i}} \psi\left(X_{i j}-\theta_{i}\right)\right]^{2}\right)=\text { minimum, } \\
\text { subject to the restraint that } \theta_{1} \leqslant \cdots \leqslant \theta_{k} .
\end{gathered}
$$

However, in view of the fact that (unlike the normal mean case), the $\psi$-function is not generally linear (though it could be piece-wise linear as in the Huber case), the computational algorithm (such as the "pool adjacent violators") discussed in Barlow et al. [1] may not be totally adaptable here. Leurgans [9] has addressed the basic issues underlying the use of the "partitionng algorithms" in the case of isotonic $M$-estimation and stressed the lack of robustness aspects. Although in our case, we have a well-defined replicated design (ensuring robustness), her study reveals the general weakness of the usual "partitioning algorithms" in robust isotonic estimation problems. On the other hand, by virtue of the Jurečková [7] linearity of $M$-statistics (with related first-order asymptotic expansions for $M$-estimators) and the asmptotic normality results discussed in the last two sections, it is possible to formulate a simple algorithm directly along the lines of Barlow et al. [1]. We shall follow this approach here.

We start with the approximate likelihood function in (3.2), and based on this reduced data set (i.e., $\hat{\boldsymbol{\theta}}_{n}$ and $n_{1}, \ldots, n_{k}$ ), we construct isotonic $M$-estimators of $\theta_{1}, \ldots, \theta_{k}$. The isotonized $M$-estimator of $\theta$, denoted by $\boldsymbol{\theta}_{n}^{*}$, is obtained by minimizing (with respect to $\theta$ )

$$
\sum_{i=1}^{k} n_{i}\left[\hat{\theta}_{i, n_{i}}-\theta_{i}\right]^{2} \quad \text { subject to } \theta_{1} \leqslant \cdots \leqslant \theta_{k} .
$$

The algorithm for the computation of $\boldsymbol{\theta}_{n}^{*}$ is the same one as for the ordered mean problem considered in detail in Section 1.2 of Barlow et al. [1]. In particular, there exist a positive integer $l: 1 \leqslant l \leqslant k$ and $l$ positive integers $k_{1}<\cdots<k_{l}=k$, such that on letting

$$
n_{j}^{*}=\sum_{i=k_{j-1}+1}^{k_{j}} n_{i} \quad \text { and } \quad \theta_{n, j}^{* *}=\sum_{i=k_{j-1}+1}^{k_{j}} n_{i} \hat{\theta}_{i, n_{i}} / n_{j}^{*}, \quad j=1, \ldots, l,
$$


we have

$$
\theta_{i, n}^{*}=\theta_{n, j}^{* *}, \quad \text { for } \quad i=k_{i-1}+1, \ldots, k_{j}, \quad j=1, \ldots, l ; \quad k_{0}=0 .
$$

Note that $l, k_{j}$, and $n_{j}^{*}$ are all stochastic in nature and they depend on the relative ordering of the basic $M$-estimators $\hat{\theta}_{1, n_{1}}, \ldots, \hat{\theta}_{k, n_{k}}$. However, there are only finitely many possible realizations for these stochastic elements. Further, note that the $\theta_{n, j}^{* *}$ for a monotone (nondecreasing) sequence while within each of the $l$ buckets, the individual $\hat{\theta}_{i, n_{i}}$ violate this monotone principle. Finally, note that the isotonic $M$-estimators are weighted linear combinations of the basic $M$-estimators, although the weights are themselves stochastic elements and depend on the relative ordering of the initial $k$ estimators. Thus we can conceive of a finite set $\Pi$ of partitions $\{\pi\}$ such that $\Pi=\bigcup\{\pi\}$ and $R^{k}$, the sample space of $\hat{\boldsymbol{\theta}}_{n}$, is the set theoretic union of disjoint sub-spaces $R_{\pi}, \pi \in \Pi$. For each $\pi \in \Pi$, there exists a matrix $\mathbf{D}_{\pi}$, such that

$$
\boldsymbol{\theta}_{n}^{*}=\mathbf{D}_{n} \hat{\boldsymbol{\theta}}_{n} \quad \text { for } \quad \hat{\boldsymbol{\theta}}_{n} \in R_{n}, \quad \forall \pi \in \Pi
$$

where the $\mathbf{D}_{\pi}$ depend on $n_{1}, \ldots, n_{k}$ through $l$ and $n_{1}^{*}, \ldots, n_{l}^{*}$ which are held fixed for the individual partitionings. $A$ a result, we may write in a compact form

$$
\boldsymbol{\theta}_{n}^{*}=\sum_{\pi \in \Pi} I\left(\hat{\boldsymbol{\theta}}_{n} \in R_{\pi}\right) \mathbf{D}_{\pi} \hat{\boldsymbol{\theta}}_{n}
$$

Incorporating (4.6), we have for every $\mathrm{x} \in R^{k}$,

$$
P\left\{n^{1 / 2}\left(\theta_{n}^{*}-\theta\right) \leqslant \mathbf{x} \mid \boldsymbol{\theta}\right\}=\sum_{\pi \in I} P\left\{n^{1 / 2}\left(\mathrm{D}_{\pi} \hat{\boldsymbol{\theta}}_{n}-\boldsymbol{\theta}\right) \leqslant \mathbf{x}, \hat{\boldsymbol{\theta}}_{n} \in R_{\pi} \mid \boldsymbol{\theta}\right\},
$$

and this form is quite amenable for further analysis. The asymptotic normality results on the classical $M$-estimators studied in earlier sections can thus be used to study the asymptotic distribution theory of isotonic $M$-estimators.

\section{The Preliminary Test Isotonic $M$-Estimator (PTIME)}

It is quite clear from (4.6) and the partitionings $R_{n}, \pi \in \Pi$, that the isotonic $M$-estimator $\theta_{n}^{*}$ may not be unbiased unless the individual $\theta_{i}$ are quite apart from each other in the domain $\theta_{1}<\cdots<\theta_{k}$. Particularly, for $\theta$ close to the line $\theta_{1}=\cdots=\theta_{k}$, the isotonic $M$-estimator may be considerably biased. For this reason, it may be quite conceivable to incorporate the preliminary test in Section 3 for constructing a PTE which should behave 
more properly for small departure of $\theta$ from the line $\theta_{1}=\cdots=\theta_{k}$ and which for large departures should behave closely to the isotonic estimator $\theta_{n}^{*}$. With this objective, we propose the following PTIME.

Corresponding to a preassigned level of significance $\alpha(0<\alpha<1)$, as in (3.29), let $c_{\alpha}^{*}$ be the critical level of the test statistic $\mathscr{L}_{n}^{*}$ in (3.25). Also, let $\tilde{\theta}_{n}=\tilde{\theta}_{n} 1$ and $\theta_{n}^{*}$ be defined as in Sections 3 and 4 . Define then

$$
\hat{\boldsymbol{\theta}}_{n}^{P T}=\tilde{\theta}_{n} I\left(\mathscr{L}_{n}^{*}<c_{\alpha}^{*}\right)+\boldsymbol{\theta}_{n}^{*} I\left(\mathscr{L}_{n}^{*} \geqslant c_{\alpha}^{*}\right) .
$$

Thus the PTIME is a convex combination of the classical and isotonic $M$-estimators of $\theta$ where the mixing coefficient is data based and rests on the preliminary test for the homogeneity of the $\theta_{i}$ against isotonic alternatives. As is generally the case with the PTE, this PTIME is not unbiased for $\theta$, even when $\theta$ deviates from the line $\theta_{1}=\cdots=\theta_{k}$. However, the relative bias of the PTIME and the isotonic $M$-estimator generally signals a clear cut preference for the PTIME. A similar picture can be obtained with respect to the risk of the two estimators with suitable quadratic error loss functions. A study of the risk of the PTIME and the isotonic ME (IME) demands the knowledge of the exact distribution theory of these estimators. Unfortunately, the distribution of the PTIME or IME is not very simple, even in the asymptotic case. Moreover, in the finite sample case, the distribution may depend on the underlying density function in a rather involved manner. For the IME or the PTIME, the main complication arises due to the distribution theory of $\boldsymbol{\theta}_{n}^{*}$ and its close relation with the preliminary test statistic $\mathscr{L}_{n}^{*}$ To obtain some meaningul results in this direction we consider some relevant asymptotic theory and use the asymptotic distributional risk measure to compare these estimates.

In the asymptotic setup of Sections 2 and 3, we assume that (2.10) holds and $n$ is large. Next, we note that if $H_{0}$ in (1.2) does not hold and $H_{1}$ holds, the test based on $\mathscr{L}_{n}^{*}$ is consistent (against any fixed alternative within the class depicted by $H_{1}$ ), and as such, by (5.1), $\hat{\theta}_{n}^{P T}$ and $\theta_{n}^{*}$ will be asymptotically equivalent, in probability. However, under $H_{0}$ or for local alternatives, this asymptotic stochastic equivalence may not hold, and hence, the relative picture becomes an important issue for closer study. For this reason, we carry out our investigation in two phases:

Phase I. Relative picture of the PTIME and IME for local alternatives and under $H_{0}$.

Phase II. Asymptotic properties of the IME for fixed alternatives.

To frame the local alternatives, we conceive of a fixed vector $\tau=\left(\tau_{1}, \ldots, \tau_{k}\right)$, such that $\tau_{1} \leqslant \cdots \leqslant \tau_{k}$, and set

$$
H_{1(n)}: \boldsymbol{\theta}=\boldsymbol{\theta}_{(n)}=\theta 1+n^{-1 / 2} \tau, \quad \theta \text { arbitrary; }
$$


by virtue of the translation equivariance of the $M$-estimators of location, we may set without any loss of generality that $\theta=0$. The null hypothesis $H_{0}$ relates to $\tau=0$. The asymptotic distribution of the unrestricted $M$-estimator (UME) $\hat{\boldsymbol{\theta}}_{n}$, given in (2.11), remains intact irrespective of any alternative (with appropriate change for $\theta$ ), but the other versions of the $M$-estimators would have different forms. For the restricted $M$-estimator (RME) $\tilde{\theta}_{n}$ in (3.4), (2.11) and (5.2) can readily be used to show that under $\left\{H_{1(n)}\right\}$,

$$
n^{1 / 2}\left(\ddot{\theta}_{n}-\theta\right) \stackrel{\mathscr{C}}{\longrightarrow} \mathcal{N}\left(\lambda^{\prime} \tau, \gamma^{-2} \sigma_{\psi}^{2}\right) .
$$

For the IME and PTIME, the asymptotic distributions are of much more complicated forms. First, we consider the case of the IME, and denote by

$$
\mathbf{D}_{\pi}^{0}=\mathbf{I} \quad \mathbf{D}_{\pi}, \quad \tau_{\pi}^{0}=\mathbf{D}_{\pi}^{0} \tau, \quad \text { for } \quad \pi \in \Pi .
$$

Then, by virtue of (4.7), we have under (5.2),

$$
\begin{aligned}
& P\left\{n^{1 / 2}\left(\boldsymbol{\theta}_{n}^{*}-\boldsymbol{\theta}_{(n)}\right) \leqslant \mathbf{x}\right\} \\
& \quad=\sum_{\pi \in \Pi} P\left\{n^{1 / 2} \mathbf{D}_{\pi}\left(\hat{\boldsymbol{\theta}}_{n}-\boldsymbol{\theta}_{(n)}\right) \leqslant \mathbf{x}+\tau_{\pi}^{0}, \hat{\boldsymbol{\theta}}_{n} \in R_{\pi} \mid \boldsymbol{\theta}_{\langle(n)}\right\} .
\end{aligned}
$$

At this stage, we may note that for each $\pi \in \Pi$,

$$
n^{1 / 2}\left[\mathbf{D}_{\pi}\left(\hat{\boldsymbol{\theta}}_{n}-\boldsymbol{\theta}_{(n)}\right)\right] \stackrel{\mathscr{Q}}{\longrightarrow} \mathcal{N}\left(\mathbf{0}, \gamma^{-2} \sigma_{\psi}^{2} \mathbf{D}_{\pi}^{\prime} \boldsymbol{\Lambda}^{-1} \mathbf{D}_{\pi}\right)
$$

However, $n^{1 / 2} \mathbf{D}_{\pi}\left(\hat{\boldsymbol{\theta}}_{n}-\boldsymbol{\theta}_{(n)}\right)$ and $n^{1 / 2}\left(\hat{\boldsymbol{\theta}}_{n}-\boldsymbol{\theta}_{(n)}\right)$ are not asymptotically independent (even under $H_{0}$ ), for every $\pi \in \Pi$. Thus, the right-hand side of (5.5) may not be factorized into two terms involving the marginal normal probabilities. Nor is $R_{\pi}$ a linear subspace of $R^{k}$ (typically, $R_{\pi}$ is a cone), and hence, $n^{1 / 2}\left(\hat{\theta}_{n}-\theta_{(n)}\right)$ may not belong to a linearly transformed form of $R_{\pi}$. On the other hand, the individual terms on the right-hand side of (5.5) can be expressed in terms of the multi-normal probability integrals (for large values of $n$ ) over specific sub-spaces in $R^{k}$, and (2.11) provides the access for this asymptotic simplification. Unfortunately, for such inequalityrestrained sub-spaces in $R^{k}$, for $k \geqslant 3$, compact forms for the probability contents based on multi-normal distributions are not available, and numerical integration seems to be a feasible way. On the other hand, by (4.6),

$$
E\left(\boldsymbol{\theta}_{n}^{*}\right)=\sum_{\pi \in \Pi} \mathbf{D}_{\pi}\left\{E\left[\hat{\boldsymbol{\theta}}_{n} I\left(\hat{\boldsymbol{\theta}}_{n} \in R_{\pi}\right)\right]\right\},
$$

so that using the fact that the components of $\hat{\boldsymbol{\theta}}_{n}$ are independent, this expectation may often be computed relatively easily. A similar simplification also holds for the second-order moments. 
Let us proceed to the case of the PTIME. First, using the asymptotic linearity results in (2.8), it follows from (3.22) through (3.26) that under $\left\{H_{1(n)}\right\}$ (as well as $H_{0}$ ), $\mathscr{L}_{n}^{*}$ in $(3.25)$ is equivalent in probability to

$$
\mathscr{L}_{n}^{0}=\gamma^{2} \sigma_{\psi}^{-2} \sum_{i=1}^{k} n_{i}\left(\theta_{i, n}^{*}-\ddot{\theta}\right)^{2},
$$

where $\widetilde{\theta}_{n}$ is defined by (3.4) and $\boldsymbol{\theta}_{n}^{*}$ is the IME of $\boldsymbol{\theta}$, defined by (4.2)-(4.4). As such, using (4.6) and (5.8), we have

$$
\mathscr{L}_{n}^{0}=\sum_{\pi \in \Pi} I\left(\hat{\boldsymbol{\theta}}_{n} \in R_{\pi}\right) n \hat{\boldsymbol{\theta}}_{n}^{\prime} \mathbf{A}_{\pi} \hat{\boldsymbol{\theta}}_{n},
$$

where

$$
\mathbf{A}_{\pi}=\left(\mathbf{D}_{\pi}^{\prime} \mathbf{\Lambda} \mathbf{D}_{\pi}-\lambda \lambda^{\prime}\right) \gamma_{2} \sigma_{\psi}^{-2}, \quad \text { for } \pi \in \Pi .
$$

Using (5.1), (5.8), (5.9), and (5.10), we may consider the following asymptotically equivalent (in probability) version of the PTIME:

$$
\begin{aligned}
\hat{\boldsymbol{\theta}}_{n}^{P T} & =\sum_{n \in \Pi} I\left(\hat{\boldsymbol{\theta}}_{n} \in R_{n}\right)\left\{\hat{\theta}_{n} 1 I\left(n \hat{\boldsymbol{\theta}}_{n}^{\prime} \mathbf{A}_{\pi} \hat{\boldsymbol{\theta}}_{n} \leqslant c_{\alpha}^{*}\right)+\mathbf{D}_{n} \hat{\boldsymbol{\theta}}_{n} I\left(n \hat{\boldsymbol{\theta}}_{n}^{\prime} \mathbf{A}_{\pi} \hat{\boldsymbol{\theta}}_{n}>c_{x}^{*}\right)\right\} \\
& =\sum_{\pi \in \Pi}\left\{I\left(\hat{\boldsymbol{\theta}}_{n} \in \boldsymbol{R}_{\pi n}^{(1)}\right)\left(\mathbf{1} \lambda^{\prime}\right) \hat{\boldsymbol{\theta}}_{n}+I\left(\hat{\boldsymbol{\theta}}_{n} \in \boldsymbol{R}_{\pi n}^{(2)}\right) \mathbf{D}_{\pi} \hat{\boldsymbol{\theta}}_{n}\right\}
\end{aligned}
$$

where

$$
\begin{aligned}
& R_{\pi n}^{(1)}=\left\{\hat{\boldsymbol{\theta}}_{n}: \hat{\boldsymbol{\theta}}_{n} \in R_{\pi} \text { and } n \hat{\boldsymbol{\theta}}_{n}^{\prime} \mathbf{A}_{\pi} \hat{\boldsymbol{\theta}}_{n} \leqslant c_{\alpha}^{*}\right\}, \\
& R_{\pi n}^{(2)}=R_{\pi} \backslash R_{\pi n}^{(1)}, \quad \pi \in \Pi .
\end{aligned}
$$

Thus, $\left\{R_{\pi n}^{(j)}, j=1,2, \pi \in \Pi\right\}$ is a finer partitioning of $R^{k}$, and we may rewrite the right-hand side of $(5.11)$ as $\sum_{\pi \in \Pi} \sum_{j=1}^{2} I\left(\hat{\boldsymbol{\theta}}_{n} \in R_{\pi n}^{(j)}\right) \mathbf{D}_{\pi}^{(j)} \hat{\boldsymbol{\theta}}_{n}$, where $\mathbf{D}_{\pi}^{(1)}=\mathbf{1} \lambda^{\prime}$ and $\mathbf{D}_{\pi}^{(2)}=\mathbf{D}_{\pi}, \pi \in \Pi$. As such, parallel to (5.5), we have under (5.2)

$$
\begin{aligned}
& \boldsymbol{P}\left\{\boldsymbol{n}^{1 / 2}\left(\hat{\boldsymbol{\theta}}_{n}^{P T}-\boldsymbol{\theta}_{(n)}\right) \leqslant \mathbf{x}\right\} \\
& \quad \simeq \sum_{\pi \subset n} \sum_{j=1}^{2} P\left\{n^{1 / 2} \mathbf{D}_{\pi}^{(j)}\left(\hat{\boldsymbol{\theta}}_{n}-\boldsymbol{\theta}_{(n)}\right) \leqslant \mathbf{x}+\tau_{\pi j}^{0}, \hat{\boldsymbol{\theta}}_{n} \in R_{\pi n}^{(j)} \mid \boldsymbol{\theta}_{(n)}\right\},
\end{aligned}
$$

and (2.11) can then be used to express (5.13) in terms of an appropriate multi-normal distribution over specific sectors of $R^{k}$; in this definition,

$$
\tau_{\pi 1}^{0}=\left(\mathbf{I}-\mathbf{D}_{\pi}^{(1)}\right) \tau \quad \text { and } \quad \tau_{\pi 2}^{0}=\tau^{0}=\mathbf{D}_{\pi} \tau, \quad \text { for } \quad \pi \in \Pi .
$$

Equation (5.7) also extends in a natural way to the case of the PTIME. 
Let us next consider the asymptotic distribution theory of IME in a relatively more general setup. Recall that the restricted alternatives we have in mind relate to $\omega^{*}$, defined in (3.1). This is a positively homogeneous cone in $R_{k}$, and the asymptotic distribution theory of the IME depends on whether $\boldsymbol{\theta}$ belongs to the interior of this cone or near any of its edges. Consider an $l$-dimensional subspace of $\omega^{*}$, where for $l$ positive integers $k_{1}<\cdots<k_{l}=k$, we have

$\theta_{k_{j-1}+1}=\cdots=\theta_{k_{j}}<\theta_{k_{j+1}}, \quad$ for $j=1, \ldots, l ; k_{0}=0 ; \quad$ and $\quad \theta_{k_{l+1}}=\infty$.

Here, $l$ is a positive integer less than or equal to $k$. It is easy to verify that when $l=k$, i.e., the $\theta_{i}$ are all distinct and ordered, as $n$ increases, the IME and classical $M$-estimator (based on the common score function) become equivalent, in probability. On the other hand, for every $l: 1 \leqslant l \leqslant k-1$, the IME and ME are not equivalent in probability, and they have different asymptotic distributions. Keeping this in mind, we would like to study the asymptotic distribution theory of the IME when $\theta$ belongs to (or lies on the boundary of) such a lower dimensional subspace of $\omega^{*}$. We may, however, note that for $\theta_{k}>\theta_{1}$, the preliminary $M$-test considered in Section 3 is consistent, and hence, the PTIME and IME would have the same asymptotic behaviour for every $l: 2 \leqslant l \leqslant k$. For $l=1$, the picture has already been drawn earlier. Thus, there is no need to bring the PTIME into this asymptotic study.

Consider a partitioning of $\{1, \ldots, k\}$ into $l$ subsets $\left[k_{j-1}+1, k_{j}\right]$, $j=1, \ldots, l$, where the $k_{j}$ are defined by $(5.15)$, and $2 \leqslant l \leqslant k-1$. We denote the centroids of the $\theta$-values within these subsets as $\theta_{(1)}^{*}, \ldots, \theta_{(i)}^{*}$, respectively. Consider then a sequence $\left\{H_{1(n)}^{*}\right\}$ of local alternatives:

$$
\begin{aligned}
& H_{1(n)}^{*}: \theta_{k_{j-1}+1+r}=\theta_{(j)}^{*}+n^{-1 / 2} \tau_{r}^{*}, \\
& \text { for } r=0, \ldots, k_{j}-k_{j-1}-1, \quad j=1, \ldots, l ;
\end{aligned}
$$

where the $\tau_{r}^{*}$ are all fixed numbers, and within each bucket, the $\tau_{r}^{*}$ are ordered. Note that by definition $\theta_{(1)}^{*}<\cdots<\theta_{(1)}^{*}$. We shall show that the asymptotic distribution of the normalized form of the IME exists and is different from that of the classical ME, for each of these local alternatives.

We denote by $\Pi_{l}$ the subset of $R^{k}$ for which

$$
\max _{r \leqslant k_{j-1}} \hat{\theta}_{r, n_{r}}<\min _{k_{j-1}<r \leqslant k_{j}} \hat{\theta}_{r, n_{r}} \leqslant \max _{k_{j-1}<r \leqslant k_{j}} \hat{\theta}_{r, n_{r}}<\min _{r>k_{j}} \hat{\theta}_{r, n_{r}}, \quad 1 \leqslant j \leqslant l,
$$

where the $\hat{\theta}_{r, n_{r}}$ are the classical $M$-estimators of the $\theta_{r}$. This subspace $\Pi_{l}$ may then be partitioned into further subsets $\pi_{l}: \pi_{l} \in \Pi_{l}$, and these are defined as in after (4.4), but restricted to $\Pi_{l}$. We then refer to (5.5) where 
$\theta_{(n)}$ now belongs to the lower dimensional space in (5.16) (actually the boundary of an $l$-dimensional subset of $\omega^{*}$ ). It is easy to show that under (5.16), $P\left\{\hat{\boldsymbol{\theta}}_{n} \in \boldsymbol{R}_{\pi} \mid \boldsymbol{\theta}_{(n)}\right\}$ converges to 0 as $n$ increases, for every $\pi$ not belonging to $\Pi_{l}$. On the other hand, for $\pi$ belonging to $\Pi_{l}$, $P\left\{n \mathbf{D}_{\pi}\left(\hat{\boldsymbol{\theta}}_{n}-\boldsymbol{\theta}_{(n)}\right) \leqslant \mathbf{x}+\tau^{* 0}, \hat{\boldsymbol{\theta}}_{n} \in R_{\pi} \mid \boldsymbol{\theta}_{(n)}\right\}$ has a nondegenerate limit, where the $\tau^{* 0}$ are defined as in (5.4) with the $\tau_{r}$ being replaced by the $\tau_{r}^{*}$. Thus, under (5.16), the asymptotic distribution function of the IME is given by

$$
\begin{aligned}
\lim _{n \rightarrow \infty} P\left\{n^{1 / 2}\left(\boldsymbol{\theta}_{n}^{*}-\boldsymbol{\theta}_{(n)}\right) \leqslant \mathbf{x} \mid(5.16)\right\} \\
\quad=\lim _{n \rightarrow \infty}\left[\sum_{\pi \in \Pi_{l}} P\left\{n^{1 / 2} \mathbf{D}_{\pi}\left(\hat{\boldsymbol{\theta}}_{n}-\boldsymbol{\theta}_{(n)}\right) \leqslant \mathbf{x}+\tau^{* 0}, \hat{\boldsymbol{\theta}}_{n} \in R_{\pi} \mid(5.16)\right\}\right] .
\end{aligned}
$$

It may be noted that for $l=1, \Pi_{l}=\Pi$ and (5.18) reduces to (5.5), while for $l \geqslant 2,(5.18)$ involves a subset of the terms appearing in (5.5), and hence, the two forms are not isomorphic. In passing, we may remark that if (5.17) holds for $l=k$ then within each of the $k$ buckets, there is only one element, and hence, $\Pi_{k}$ consists of the cone $\hat{\theta}_{1, n_{1}} \leqslant \cdots \leqslant \hat{\theta}_{k, n_{k}}$. As such, (4.5) holds with $\mathbf{D}_{\pi}=\mathbf{I}$ with probability converging to 1 as $n \rightarrow \infty$. Thus, in this case, the classical $M$-estimator and the IME based on the same score function becomes asymptotically equivalent, in probability. Thus, (2.11) applies to the IME as well.

It is quite clear that the computation of the exact bias and mean product matrix of the IME and PTIME is highly involved; even the asymptotic case is not that simple to handle. For small values of $k$ (viz., $k=3,4$, etc.), term by term evaluation of $(5.5)$ or (5.18) is possible, although the task becomes prohibitively laborious as $k$ increases. For this reason, we take recourse to simulation studies of the relative bias and efficiency of the PTIME and IME. In this context, we interpret the relative efficiency $\left(e^{*}\right)$ of the PTIME with respect to the IME in the usual way as the inverse ratio of the generalized variance of their respective asymptotic distributions.

\section{Some Simulation Studies}

We consider specifically the case of three samples (i.e., $k=3$ ) and for the $M$-estimators of location, we choose the Huber score function with $K=1.5$, i.e., we take

$$
\psi(x)= \begin{cases}x, & |x| \leqslant K=1.5 \\ K \operatorname{sign} x, & |x|>K\end{cases}
$$

All the samples are generated by random normal deviates with appropriate shifts in the location parameters. Since the $M$-estimators are translation- 
TABLE I

Asymptotic Bias and Asymptotic Relative Efliciency of the PTE and IME under $H_{0}$

\begin{tabular}{|c|c|c|c|c|c|c|c|}
\hline \multirow[b]{3}{*}{$n$} & \multicolumn{6}{|c|}{ Bias } & \multirow{3}{*}{$\begin{array}{c}\text { Relative } \\
\text { efficiency } \\
e^{*}\end{array}$} \\
\hline & \multicolumn{3}{|c|}{$\begin{array}{c}\text { PTIME } \\
\text { Component }\end{array}$} & \multicolumn{3}{|c|}{$\begin{array}{c}\text { IME } \\
\text { Component }\end{array}$} & \\
\hline & 1 & 2 & 3 & 1 & 2 & 3 & \\
\hline 10 & 0.0061 & 0.0136 & 0.0329 & -0.1099 & 0.0058 & 0.1318 & 4.3311 \\
\hline 15 & 0.0069 & 0.0141 & 0.0251 & -0.0931 & 0.0074 & 0.1071 & 4.9139 \\
\hline 20 & 0.0070 & 0.0130 & 0.0250 & -0.0795 & 0.0068 & 0.0955 & 4.2977 \\
\hline 25 & 0.0039 & 0.0094 & 0.0220 & -0.0748 & 0.0021 & 0.0848 & 4.7852 \\
\hline 30 & 0.0057 & 0.0095 & 0.0195 & -0.0685 & 0.0043 & 0.0776 & 5.4195 \\
\hline 35 & 0.0063 & 0.0103 & 0.0209 & -0.0601 & 0.0053 & 0.0727 & 5.0517 \\
\hline 40 & 0.0063 & 0.0101 & 0.0180 & -0.0566 & 0.0062 & 0.0692 & 4.7341 \\
\hline
\end{tabular}

equivariant, we have taken the location parameter of the first distribution as 0 . All the three samples are taken to be of equal size $(n)$ and various combinations of $n$ and possibly uneven spacings of the location parameters. Tables I-VII pertain to the simulation results on the bias and relative efficiency $\left(e^{*}\right)$ of the PTE and IME.

Recall that here $n$ stands for the (equal) individual sample sizes, so that the combined sample size is $3 n$. It is clear from Tables I and II that under the null hypothesis $H_{0}$ or for small departures from $H_{0}$, the PTIME performs better than the IME both in terms of the bias and mean product

TABLE II

Same Entries for $\theta-(0,0.1,0.2)$ (i.e., Equally Spaced Means)

\begin{tabular}{|c|c|c|c|c|c|c|c|}
\hline \multirow[b]{3}{*}{$n$} & \multicolumn{6}{|c|}{ Bias } & \multirow{3}{*}{$\begin{array}{l}\text { Relative } \\
\text { efficiency } \\
e^{*}\end{array}$} \\
\hline & \multicolumn{3}{|c|}{$\begin{array}{l}\text { PTIME } \\
\text { Component }\end{array}$} & \multicolumn{3}{|c|}{$\begin{array}{c}\text { IME } \\
\text { Component }\end{array}$} & \\
\hline & 1 & 2 & 3 & 1 & 2 & 3 & \\
\hline 10 & 0.0894 & 0.0111 & -0.0428 & -0.0704 & 0.0052 & 0.0928 & 2.0132 \\
\hline 15 & 0.0822 & 0.0138 & -0.0449 & -0.0542 & 0.0068 & 0.0689 & 1.7756 \\
\hline 20 & 0.0799 & 0.0119 & -0.0421 & -0.0425 & 0.0064 & 0.0588 & 1.5676 \\
\hline 25 & 0.0749 & 0.0066 & -0.0420 & -0.0382 & 0.0046 & 0.0486 & 1.4826 \\
\hline 30 & 0.0750 & 0.0064 & -0.0439 & -0.0338 & 0.0039 & 0.0433 & 1.1027 \\
\hline 35 & 0.0740 & 0.0078 & -0.0425 & -0.0270 & 0.0052 & 0.0396 & 1.3054 \\
\hline 40 & 0.0717 & 0.0077 & -0.0438 & -0.0237 & 0.0054 & 0.0371 & 1.2109 \\
\hline
\end{tabular}


TABLE III

Average Bias and Relative Efficiency for $\theta=(0,0.05,0.15)$ (Uneven Spacing)

\begin{tabular}{|c|c|c|c|c|c|c|c|}
\hline \multirow[b]{3}{*}{$n$} & \multicolumn{6}{|c|}{ Bias } & \multirow{3}{*}{$\begin{array}{l}\text { Relative } \\
\text { efficiency } \\
e^{*}\end{array}$} \\
\hline & \multicolumn{3}{|c|}{$\begin{array}{c}\text { PTIME } \\
\text { Component }\end{array}$} & \multicolumn{3}{|c|}{$\begin{array}{c}\text { IME } \\
\text { Component }\end{array}$} & \\
\hline & 1 & 2 & 3 & 1 & 2 & 3 & \\
\hline 10 & 0.0636 & 0.0287 & -0.0305 & -0.0844 & 0.0158 & 0.0963 & 2.5935 \\
\hline 15 & 0.0615 & 0.0308 & -0.0376 & -0.0678 & 0.0170 & 0.0723 & 2.3186 \\
\hline 20 & 0.0614 & 0.0278 & -0.0376 & -0.0554 & 0.0165 & 0.0616 & 2.1086 \\
\hline 25 & 0.0558 & 0.0235 & -0.0345 & -0.0506 & 0.0113 & 0.0513 & 2.0008 \\
\hline 30 & 0.0580 & 0.0230 & -0.0376 & -0.0456 & 0.0132 & 0.0459 & 1.9718 \\
\hline 35 & 0.0579 & 0.0237 & -0.0365 & -0.0381 & 0.0142 & 0.0418 & 1.8193 \\
\hline 40 & 0.0565 & 0.0236 & -0.0380 & -0.0347 & 0.0144 & 0.0391 & 1.6909 \\
\hline
\end{tabular}

TABLE IV

Same Entries for $\theta=(0.0 .2,0.5)$ (i.e., Uneven Spacings)

\begin{tabular}{|c|c|c|c|c|c|c|c|}
\hline \multirow[b]{3}{*}{$n$} & \multicolumn{6}{|c|}{ Bias } & \multirow{3}{*}{$\begin{array}{l}\text { Relative } \\
\text { efficiency } \\
e^{*}\end{array}$} \\
\hline & \multicolumn{3}{|c|}{$\begin{array}{l}\text { PTIME } \\
\text { Component }\end{array}$} & \multicolumn{3}{|c|}{$\begin{array}{c}\text { IME } \\
\text { Component }\end{array}$} & \\
\hline & 1 & 2 & 3 & 1 & 2 & 3 & \\
\hline 10 & 0.1672 & 0.0325 & -0.1174 & -0.0395 & 0.0186 & 0.0485 & 1.2090 \\
\hline 15 & 0.1446 & 0.0333 & -0.1081 & -0.0261 & 0.0182 & 0.0295 & 1.0649 \\
\hline 20 & 0.1265 & 0.0293 & -0.0906 & -0.0188 & 0.0160 & 0.0256 & 0.9652 \\
\hline 25 & 0.1103 & 0.0208 & -0.0791 & -0.0163 & 0.0093 & 0.0191 & 0.9274 \\
\hline 30 & 0.0905 & 0.0223 & -0.0641 & -0.0153 & 0.0113 & 0.0174 & 0.7065 \\
\hline 35 & 0.0671 & 0.0186 & -0.0419 & -0.0076 & 0.0107 & 0.0156 & 0.8390 \\
\hline
\end{tabular}

TABLE $V$

Same Entries for $\boldsymbol{\theta}=(0,0.5,1.0)$ (i.e., Large Equal Spacing)

\begin{tabular}{|c|c|c|c|c|c|c|c|}
\hline \multirow[b]{3}{*}{$n$} & \multicolumn{6}{|c|}{ Bias } & \multirow{3}{*}{$\begin{array}{c}\text { Relative } \\
\text { efficiency } \\
e^{*}\end{array}$} \\
\hline & \multicolumn{3}{|c|}{$\begin{array}{l}\text { PTIME } \\
\text { Component }\end{array}$} & \multicolumn{3}{|c|}{$\begin{array}{c}\text { IME } \\
\text { Component }\end{array}$} & \\
\hline & 1 & 2 & 3 & 1 & 2 & 3 & \\
\hline 10 & 0.1315 & 0.0084 & -0.1045 & -0.0019 & 0.0015 & 0.0280 & 0.7553 \\
\hline 15 & 0.0756 & 0.0072 & -0.0572 & 0.0031 & 0.0041 & 0.0143 & 0.7620 \\
\hline 20 & 0.0387 & 0.0052 & -0.0194 & 0.0045 & 0.0043 & 0.0139 & 0.7978 \\
\hline 25 & 0.0211 & 0.0002 & -0.0071 & 0.0013 & -0.0012 & 0.0119 & 0.8278 \\
\hline 30 & 0.0087 & 0.0039 & 0.0033 & -0.0001 & 0.0023 & 0.0111 & 0.8743 \\
\hline 35 & 0.0091 & 0.0037 & 0.0071 & 0.0037 & 0.0030 & 0.0112 & 0.9082 \\
\hline 40 & 0.0063 & 0.0048 & 0.0095 & 0.0028 & 0.0043 & 0.0116 & 0.9499 \\
\hline
\end{tabular}


TABLE VI

Average Bias and Relative Efficiency for $\theta=(0,0.2,0,8)$

(Uncven Large Spacings)

\begin{tabular}{|c|c|c|c|c|c|c|c|}
\hline \multirow[b]{3}{*}{$n$} & \multicolumn{6}{|c|}{ Bias } & \multirow{3}{*}{$\begin{array}{c}\text { Relative } \\
\text { efficiency } \\
e^{*}\end{array}$} \\
\hline & \multicolumn{3}{|c|}{$\begin{array}{c}\text { PTIME } \\
\text { Component }\end{array}$} & \multicolumn{3}{|c|}{$\begin{array}{c}\text { IME } \\
\text { Component }\end{array}$} & \\
\hline & 1 & 2 & 3 & 1 & 2 & 3 & \\
\hline 10 & 0.1379 & 0.0766 & -0.1431 & -0.0366 & 0.0404 & 0.0238 & 0.9315 \\
\hline 15 & 0.0933 & 0.0597 & -0.0978 & -0.0241 & 0.0340 & 0.0117 & 0.8735 \\
\hline 20 & 0.0546 & 0.0446 & -0.0538 & -0.0177 & 0.0283 & 0.0121 & 0.8454 \\
\hline 25 & 0.0314 & 0.0302 & -0.0311 & -0.0159 & 0.0174 & 0.0106 & 0.8580 \\
\hline 30 & 0.0163 & 0.0259 & -0.0137 & -0.0149 & 0.0179 & 0.0104 & 0.8732 \\
\hline 35 & 0.0118 & 0.0216 & -0.0044 & -0.0089 & 0.0161 & 0.0107 & 0.8947 \\
\hline 40 & 0.0066 & 0.0194 & -0.0008 & -0.0075 & 0.0149 & 0.0113 & 0.8940 \\
\hline
\end{tabular}

matrix-risk. Also, the bias of the PTIME and IME are not in concordance with each other. A somewhat diferent picture emerges in the uneven spacing case and for alternatives not so close to the null one. The last three tables indicate the superiority of the IME to PTIME. This is not surprising: We have both uneven spacings and moderate deviations from the null hypothesis. Thus, for alternatives close to the null hypothesis (of the homogeneity of the $\theta_{i}$ ), the PTIME performs better than the IME, while the opposite picture hols when $\theta$ moves away from the line of homogeneity. In any case, if $\theta$ is too far away from this line, the PTIME and IME both

TABLE VII

Same Entries for $\boldsymbol{\theta}=(0,0.5,1.5)$ (i.e., Large Uneven Spacings)

\begin{tabular}{|c|c|c|c|c|c|c|c|}
\hline \multirow[b]{3}{*}{$n$} & \multicolumn{6}{|c|}{ Bias } & \multirow{3}{*}{$\begin{array}{l}\text { Relative } \\
\text { efficiency } \\
e^{*}\end{array}$} \\
\hline & \multicolumn{3}{|c|}{$\begin{array}{c}\text { PTIME } \\
\text { Component }\end{array}$} & \multicolumn{3}{|c|}{$\begin{array}{c}\text { IME } \\
\text { Component }\end{array}$} & \\
\hline & 1 & 2 & 3 & 1 & 2 & 3 & \\
\hline 10 & 0.0349 & 0.0187 & -0.0182 & -0.0018 & 0.0128 & 0.0166 & 0.8411 \\
\hline 15 & 0.0126 & 0.0120 & 0.0010 & 0.0031 & 0.0099 & 0.0085 & 0.9319 \\
\hline 20 & 0.0077 & 0.0086 & 0.0082 & 0.0045 & 0.0073 & 0.0110 & 0.9615 \\
\hline 25 & 0.0026 & 0.0019 & 0.0094 & 0.0013 & 0.0011 & 0.0096 & 0.9727 \\
\hline 30 & 0.0006 & 0.0050 & 0.0103 & -0.0001 & 0.0035 & 0.0100 & 0.9629 \\
\hline 35 & 0.0043 & 0.0045 & 0.0111 & 0.0037 & 0.0036 & 0.0105 & 0.9717 \\
\hline 40 & 0.0038 & 0.0054 & 0.0113 & 0.0028 & 0.0046 & 0.0113 & 0.9596 \\
\hline
\end{tabular}


perform very similarly. Moreover, the PTIME is never too inefficient relative to the IME, although it can be considerably more efficient (see Table I). Thus, the PTIME can be posed as an efficiency-robust competitor of the usual IME. For some further numerical studies, we refer to Karmous [8].

\section{ACKNOWLEDGMENTS}

We are grateful to Professor Vernon M. Chinchilli for his most critical reading of the manuscript leading to various improvements.

\section{REFERENCES}

[1] Barlow, R. E., Bartholomew, D. J., Bremner, J. M., and Brunk, H. D. (1972). Statistical Inference under Order Restrictions. Wiley, New York.

[2] Boyd, M. N., AND SEN, P. K. (1983). Union-intersection rank tests for ordered alternatives in some simple linear models. Comm. Statist.-Theory Methods 12 1737-1758.

[3] Chinchilli, V. M., And Sen, P. K. (1981) Multivariate linear rank statistics and the union-intersection principle for hypothesis testing under restricted alternatives. Sankhyā Ser. B 43 135-151.

[4] Chinchilli, V. M., ANd Sen, P. K. (1981). Multivariate linear rank statistics and the union-intersection principle for the orthant restriction problem. Sankhyā Ser. B 43 152-171.

[5] DE, N. (1975). Rank tests for randomized blocks against ordered alternatives. Calcutta Statist. Assoc. Bull. 25 1-27.

[6] Gupta, S. S. (1963). Probability integrals of multivariate normal and multivariate $t$. Ann. Math. Statist. 34 792-838.

[7] JuREČKoví, J. (1977). Asymptotic relations of $M$-estimates and $R$-estimates in linear regression models. Ann. Statist. 5 464-472.

[8] Karmous, A. R. (1986). Robust, Isotonic and Preliminary Test Estimators in Some Linear Models under Rrestraints. Doctoral dissertation. University North Carolina, Chapel Hill, NC.

[9] Leurgans, S. (1986). Isotonic $M$-estimation. In Advances in Order Restricted Statistical Inference (R. Dykstra, T. Robertson, and F. T. Wright, Eds.), pp. 48-68, SpringerVerlag, New York.

[10] Sen, P. K., and Saleh, A. K. M. E. (1987). On preliminary test and shrinkage $M$-estimation in linear models. Ann. Statist. 15 1580-1592.

[11] Singer, J. M., AND SEN, P. K. (1985). $M$-methods in multivariate linear models. J. Multivariate Anal. 17 168-184.

[12] Tsai, M.-T. M., AND SEN, P. K. (1987). Asymptotic distribution of UI-LMPR tests for restricted alternatives. Submitted for publication. 